\title{
Neonatal Hypoglycemia Related to Glycine Levels in Uncontrolled Gestational Diabetes Mellitus during Mid-Late Pregnancy: Multicenter, Prospective Case-Cohort Observational Study
}

\author{
Yuhang Long $\left(\mathbb{D},{ }^{1,2}\right.$ Le Chen, ${ }^{1}$ Yaochao Yang, ${ }^{1,2}$ Wei Liu, ${ }^{3}$ Hanxi Zhang, ${ }^{4}$ Xin Bao, ${ }^{5}$ \\ Meijun Liu, ${ }^{6}$ WuJuan Chen, ${ }^{1}$ Fangfang Jiang, ${ }^{1}$ Ting Hong, ${ }^{1}$ Jianying Wang, ${ }^{1}$ Yao Chen, \\ Jianyong Cao, ${ }^{1}$ Dan Xie, ${ }^{1,2}$ and Zhaojuan Su $\mathbb{D D}^{1,2}$ \\ ${ }^{1}$ Department of Gynecology and Obstetrics, The Fifth Affiliated Hospital of Sun Yat-sen University, Zhuhai, \\ Guangdong 519000, China \\ ${ }^{2}$ Guangdong Provincial Key Laboratory of Biomedical Imaging, The Fifth Affiliated Hospital, Sun Yat-sen University, Zhuhai, \\ Guangdong Province 519000, China \\ ${ }^{3}$ Department of Clinical Laboratory, The Fifth Affiliated Hospital of Sun Yat-sen University, Zhuhai, Guangdong 519000, China \\ ${ }^{4}$ National Center for AIDS/STD Control and Prevention, China CDC, Beijing 102206, China \\ ${ }^{5}$ Department of Gynecology and Obstetrics, Zhuhai Center for Maternal and Child Health Care, Zhuhai, Guangdong 519000, China \\ ${ }^{6}$ Department of Gynecology and Obstetrics, Zhuhai People's Hospital, Zhuhai, Guangdong 519000, China
}

Correspondence should be addressed to Zhaojuan Su; 2847066683@qq.com

Received 16 July 2020; Revised 29 September 2020; Accepted 30 October 2020; Published 21 November 2020

Academic Editor: Riccardo Calafiore

Copyright (C) 2020 Yuhang Long et al. This is an open access article distributed under the Creative Commons Attribution License, which permits unrestricted use, distribution, and reproduction in any medium, provided the original work is properly cited.

\begin{abstract}
Aims. To explore the relationship between gestational diabetes mellitus (GDM) and neonatal cord blood amino acid and carnitine levels after GDM was diagnosed among pregnant women monitoring glycosylated haemoglobin levels of 5.5\%-6.4\% during midlate gestation. Methods. In all, 7289 qualified participants were recruited and divided into two groups (GDM and control groups) between 1 July 2015 and 1 July 2020, and all maternal-neonatal data were collected and analyzed at three centers. Results. Interestingly, glycine in cord blood was not only significantly different between groups $(15.52$ vs. $6.67, P<0.001)$ but also associated with neonatal hypoglycemia $(r=0.132, P<0.001)$. Although glycine was an independent positive factor with neonatal hypoglycemia, it had lacked effective size to predict the risk of neonatal hypoglycemia $(b=0.002, P<0.001)$. Conclusion. The study identifies some differences and relationships in maternal-neonatal data when the GDM group has fluctuating glycosylated haemoglobin levels of 5.5\%-6.4\% without hypoglycemic drug intervention, compared with the control group. Although umbilical cord blood of glycine levels has a lack of effective power to predict the risk of neonatal hypoglycemia, it is probably an independent factor involved in the maternal-neonatal glucolipid metabolism.
\end{abstract}

\section{Introduction}

Maternal disorder of glucose metabolism probably affected maternal-neonatal health and metabolism during pregnancy $[1,2]$. Meanwhile, on the basis of the International Association of Diabetes and Pregnancy Study Groups (IADPSG), over $17 \%$ of pregnant women would be diagnosed with gestational diabetes mellitus [3]. Therefore, GDM could be a common disturbance of carbohydrate metabolism in pregnant women and caused adverse pregnancy outcomes, such as maternal preeclampsia and neonatal hypoglycemia according to the Hyperglycemia and Adverse Pregnancy Outcomes (HAPO) study [4].

All pregnant women in GDM would accept the dietary therapy and physical exercise treatment after the strict diagnosis standard of OGTT according to IADPSG. Additionally, glycosylated haemoglobin (HbAlc) levels were widely used for diabetic control. Some pregnant women were poor with the glucose regulation control based on the IADPSG recommendation range of $\mathrm{HbA1c}$ levels below 5.5\% during mid- 
late pregnancy. Meanwhile, those pregnant women in GDM were probably reluctant to accept drugs such as insulin even for serious uncontrolled hyperglycemia.

According to IADPSG and American Diabetes Association (ADA), HbA1c levels could be a useful and effective evaluation to blood glucose control, but it was still an argument as to what range was appropriate and what therapeutic schedule should be used on a critical range. It was considered that worse glucose control as HbA1c levels between 5.5\% and $6.4 \%$ among pregnant women with GDM $\$ and it was unnecessary for further treatment, immediately. In China and India, most pregnant women were recommended to follow IADPSG because of racial and economic factors [5].

Pregnancy complicated with diabetes, with out of control blood glucose levels as an obvious dysglycemia, it was associated with a highly increased risk of adverse pregnancy outcomes, such as neonatal hypoglycemia [6, 7]. If pregnant women in GDM appeared with HbA1c levels $\geq 6.5 \%$, it also was diagnosed as pregestational diabetes mellitus (PGDM) following the standard of IADPSG. Insulin as a first-line treatment should be considered among those pregnant women. However, mid-late pregnancy maternal blood glucose monitoring by HbAlc fluctuated in 5.5\%-6.4\% which was an unclear and potentially risky range, especially for those pregnant women in GDM.

Some recent studies reported that maternal-neonatal metabolites were correlated with insulin resistance and diabetes $[8,9]$. For example, fetal carbohydrates [10], lipid profiles [11], carnitines [12], and amino acids [13], particularly branched-chain amino acids [14] and aromatic amino acids [15], could be influenced by GDM. However, little articles reported the change and relationship of neonatal amino acids and carnitines when maternal consistent dysglycemia because of the failure of glucose control during pregnancy.

On the other hand, maternal glucose homeostasis tended to remain elevated because placental hormones and maternal insulin resistance occurred particularly in mid-late pregnancy $[16,17]$, but it was still unclear. Maternal dysglycemia had been proven to be a contribution for fetal macrosomia and neonatal hypoglycemia [18]. However, the exact effect of how maternal glycolipid dysfunction influenced neonatal metabolism in offspring remained unclear because it was rarely reported and early interfered by researchers. In the study, we carried out a prospective observational study to explore the associations between GDM in dysfunction status and neonatal amino acids and carnitines when GDM monitored by $\mathrm{HbA1c}$ average levels was between $5.5 \%$ and $6.4 \%$.

Therefore, the first research shows a rigorous accurate study on the part of maternal-neonatal metabolism with GDM ranged $\mathrm{HbA1c}$ average levels between $5.5 \%$ and $6.4 \%$ because of the elimination of drug interference and the safety of pregnancy. There was also a further study on the argument of the failure of controlling glucose when HbAlc average levels were between $5.5 \%$ and $6.4 \%$ during mid-late pregnancy and maternal-neonatal metabolism and outcomes. It may provide effective evidence for the prevention of GDM and adverse maternal-neonatal outcomes and a new perspective for understanding the mechanism of maternal and neonatal metabolism.

\section{Materials and Methods}

2.1. Ethics Statement. The present study had been approved by the ethical committees of the Fifth Affiliated Hospital of Sun Yat-sen University, Zhuhai People's Hospital, and Zhuhai Center for Maternal and Child Health Care, and all participants provided written informed consent.

All subjects were informed that they would be followed up and collected blood sample. It was none of interference and difference compared with daily clinical advice and treatment in the follow-up.

2.2. Sample Size. According to the multicenter prospective cohort study of 1813 pregnant women, the OR rate of neonatal hypoglycemia (OR 4.86 [95\% CI 2.04, 11.53]) was used to determine the sample size [19]. We estimated that 1107 patients per group would be required to find the morbidity of hypoglycemia in neonates approximately being $3 \%$ in the control group using two tailed $\alpha=0.05$ and one-sided $\beta=$ 0.10 and the power of test $=0.90$. We increased the study size to 1200 per group to allow for possible dropouts or patient loss as a result of a clinical situation.

2.3. Study Design. All participants who delivered in our centers between 1 July 2015 and 1 July 2020 were willing to engage in the follow-up, test HbAlc levels each month at least three times and donate maternal-neonatal blood sample during mid-late pregnancy. Meanwhile, with more and more large-scale multicenter clinical research studies, the importance of quality management was needed. Those hospitals were the third grade hospitals which had the same clinical protocol for follow-up and collected all blood samples to the key national laboratory for storage and analyzation.

First, potential participants that meet the inclusion criteria would be invited by research assistants, and the objective, procedure, benefits, and risks of the study would be explained. Pregnant women would be invited to participate in this study if they were between 18 and 40 years of age and had had their OGTT in our centers before 24-28 weeks of gestation.

There was a clear recommendation to screen for GDM in high-risk pregnant women using the IADPSG and ADA criteria, and thus, pregnant women would be excluded from the study if they had any of the following risk factors or conditions: (1) history of GDM or preexisting diabetes mellitus (DM); (2) family history of DM (first-degree relative with diabetes or a sister with GDM); (3) body mass index (BMI) $>30 \mathrm{~kg} / \mathrm{m}^{2}$ before pregnancy; (4) previous macrosomia (baby with birth weight $>4000 \mathrm{~g}$ ) or a history of stillbirth; (5) polycystic ovary syndrome; (6) medications: corticosteroids, antipsychotics, and drugs influencing glycolipids such as statins; (7) participant not willing to take OGTT at 24-28 gestational weeks and $\mathrm{HbA1c}$ levels each month until labor (less three times), not willing to have a series of prenatal care visits and delivery, or refusing blood sample collection during mid-late pregnancy.

2.4. Diagnosis Standards and Medical Care. The diagnosis of GDM was based on a $75 \mathrm{~g}$ oral glucose tolerance test (OGTT) performed between 24 and 28 gestational weeks, according to 


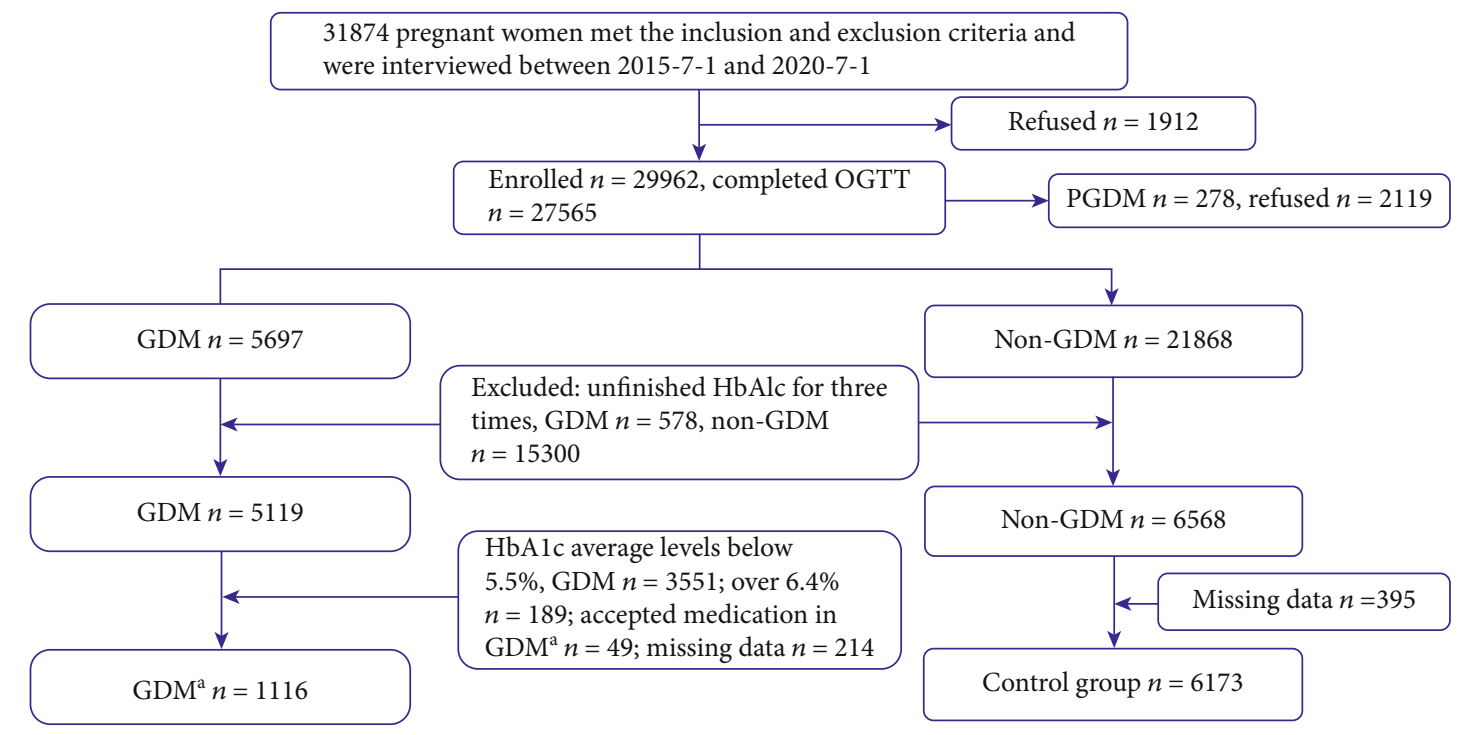

FIGURE 1: Subjects' flowchart. It shows a series of details on the follow-up. 7289 pregnant women completely finished the follow-up between 2015-7-1 and 2020-7-1. HbA1c test each month with missing details: (1) 24-28 weeks at the first time GDM $n=0$, non-GDM $n=654$; (2) both 28-32 weeks and 32-36 weeks GDM $n=0$, non-GDM $n=12924$; (3) before the delivery GDM $n=0$, non-GDM $n=1722$. Missing data included out of the delivery in three centers: GDM $n=159$, non-GDM $n=195$; incomplete clinical data: GDM $n=12$, non-GDM $n=18$ ;blood sample problems: GDM $n=34$, non-GDM $n=171$; or rejected to follow: GDM $n=9$, non-GDM $n=11$. PGDM: pregestational diabetes mellitus; GDM: gestational diabetes mellitus; HbA1c (\%): glycated haemoglobin. ${ }^{\mathrm{a}}$ Glycosylated haemoglobin monitoring range is 5.5-6.4\%.

IADPSG criteria (fasting $\geq 5.1 \mathrm{mmol} / \mathrm{L}, 1 \mathrm{~h} \geq 10.0 \mathrm{mmol} / \mathrm{L}$, and $2 \mathrm{~h} \geq 8.5 \mathrm{mmol} / \mathrm{L}$ ). Recruited subjects accepted the standard of treatment for GDM.

The diagnosis of neonatal hypoglycemia was based on a fingertip blood glucose test after labor within 5 minutes, according to the American Academy of Pediatrics criteria (fingertip blood glucose $<2.22 \mathrm{mmol} / \mathrm{L}$ ) [20].

2.5. Blood Sample Collection. In brief, all pregnant women had blood tests from the prenatal examination or hospitalization examination before their delivery. All neonates included in our study had amino acids and carnitines measured in dried blood specimens collected from umbilical cord blood after the second stage within 10 minutes.

2.6. Study Clinical Test. All subjects took blood tests including OGTT, HbAlc, and lipid tests such as triglycerides (TG), total cholesterol (TC), high-density lipoproteincholesterol (HDL-C), and low-density lipoproteincholesterol (LDL-C).

Blood samples in the anticoagulative tubes for measuring glycated haemoglobin, lipid profiles, and glucose in plasma were sent to the clinical laboratory within 1 hour of collection.

Maternal glucose levels were measured using a GODPAP kit (Human, Shanghai, China). Glycated haemoglobin was tested by an ADAMS ${ }^{\mathrm{TM}}$ A1c HA-8180 kit (Arkray USA Inc., Kyoto, Japan) and based on the standardization of the National Glycohemoglobin Standardization Program and Diabetes Control and Complications Trial. Triglyceride (TG) levels were measured using a glycerol phosphate oxidase test kit (Shanghai, China). Total cholesterol (TC) levels were measured using an oxidase test kit (Human, Shanghai, China). High-density lipoprotein-cholesterol (HDL-C) and low-density lipoprotein-cholesterol (LDL-C) levels were determined by direct method kits (Human, Shanghai, China).

2.7. Laboratory Measurements. Neonatal blood was collected and analyzed for 17 types of amino acids and 30 types of carnitines after the delivery of the placenta.

A single $3.2 \mathrm{~mm}$ diameter dried newborn's blood disc per sample was punched from the dried blood spot card and extracted with $90 \mu \mathrm{L}$ of methanol (including internal standards from Cambridge Isotope Inc.) at $30^{\circ} \mathrm{C}$ for 30 minutes. After centrifugation at $2000 \mathrm{~g}$ for 5 minutes, $50 \mu \mathrm{L}$ of supernatant was transferred and nitrogen-dried at room temperature, followed by addition of $50 \mu \mathrm{L}$ of derivatization reagent (acetyl chloride: butanol $(v: v)=1: 9)$, incubation at $60^{\circ} \mathrm{C}$ for 30 minutes, nitrogen-drying, and reconstitution with $75 \mu \mathrm{L}$ of $80 \%$ acetonitrile $/ \mathrm{H}_{2} \mathrm{O}(v / v)$. After centrifugation at $4000 \mathrm{~g}$ for 15 minutes, $50 \mu \mathrm{L}$ of supernatant was taken for liquid chromatographic-mass spectrometric (LC-MS) analysis.

An Acquity UPLC I-Class Xevo TQD mass spectrometer (Waters) was used to measure the concentration levels of multiple amino acids and carnitines in the dried blood spot with the application of direct flow injection and multiple reaction monitoring (MRM). The sample was introduced into the electrospray ionization interface by the mobile phase of $80 \%$ acetonitrile $/ \mathrm{H}_{2} \mathrm{O}(v / v)$ with scheduled flow rates. The mass spectrometer was tuned as recommended by the manufacturer with resolution, capillary voltage, source temperature, and desolvation temperature set at $0.7 \mathrm{Da}, 3.5 \mathrm{kV}$, $120^{\circ} \mathrm{C}$, and $350^{\circ} \mathrm{C}$, respectively. All the targeted analytes were 
TABLE 1: Characteristics and outcomes of the study population.

\begin{tabular}{|c|c|c|c|c|}
\hline & GDM & Control & $\chi^{2}$ & $P$ \\
\hline$N$ & 1116 & 6173 & & \\
\hline Maternal age (years) & $29.6 \pm 3.56$ & $28.32 \pm 3.00$ & - & $<0.001$ \\
\hline Gestational weeks (days) & $267.08 \pm 10.82$ & $274.87 \pm 12.23$ & - & $<0.001$ \\
\hline \multicolumn{5}{|l|}{ Body mass index $\left(\mathrm{kg} / \mathrm{m}^{2}\right)$} \\
\hline Prepregnancy & $23.19 \pm 3.33$ & $23.21 \pm 3.27$ & - & 0.723 \\
\hline Antepartum & $26.25 \pm 3.46$ & $27.14 \pm 3.56$ & - & $<0.001$ \\
\hline Mode of delivery, no. (\%) & & & 5.974 & 0.113 \\
\hline Spontaneous vaginal & $722(64.7)$ & $4074(66.0)$ & & \\
\hline Assisted vaginal & $93(8.3)$ & $395(6.4)$ & & \\
\hline Elective cesarean & $172(15.4)$ & $944(15.3)$ & & \\
\hline Emergency cesarean & $129(11.6)$ & $760(12.3)$ & & \\
\hline \multicolumn{5}{|l|}{ Neonatal outcomes } \\
\hline Gender, no. (\%) & & & 0.008 & 0.931 \\
\hline Male & $581(52.1)$ & $3205(51.9)$ & & \\
\hline Female & $535(47.9)$ & $2968(48.1)$ & & \\
\hline Birth weight (g) & $3242.49 \pm 456.30$ & $3198.92 \pm 449.14$ & - & 0.004 \\
\hline Hypoglycemia, no. (\%) & $145(13.4)$ & $123(2.0)$ & 322.926 & $<0.001$ \\
\hline Premature, no. (\%) & $166(14.9)$ & $362(5.7)$ & 114.199 & $<0.001$ \\
\hline \multicolumn{3}{|c|}{ Meconium-stained amniotic fluid, no. (\%) } & 5.677 & 0.128 \\
\hline Clear & $812(72.8)$ & $4432(71.8)$ & & \\
\hline I & $218(19.5)$ & $1346(21.8)$ & & \\
\hline II & $65(5.8)$ & $315(5.1)$ & & \\
\hline III & $21(1.9)$ & $80(1.3)$ & & \\
\hline \multicolumn{5}{|l|}{ Bad outcomes after delivery, no. (\%) } \\
\hline Apgar $\leq 7$ at $1 \mathrm{~min}$ & $36(3.2)$ & $278(4.5)$ & 3.743 & 0.053 \\
\hline Apgar $\leq 7$ at $5 \mathrm{~min}$ & $7(0.6)$ & $48(0.8)$ & 0.285 & 0.593 \\
\hline Apgar $\leq 7$ at $10 \mathrm{~min}$ & $7(0.6)$ & $40(0.6)$ & 0.006 & 0.936 \\
\hline Admission to intensive care unit & $9(0.8)$ & $56(0.9)$ & 0.108 & 0.742 \\
\hline
\end{tabular}

Data given as the mean \pm SD or no. (\%). GDM: gestational diabetes mellitus; BMI: body mass index.

quantitatively measured according to the ion pair transitions of both their own and corresponding internal standards. The MassLynx NT 4.1 Software Suite was used to control the instrument and for data processing and analysis.

2.8. Clinical Data. Clinical data, including age, gestational weeks, BMI, mode of delivery, fetal birth weight, and maternal-neonatal outcomes, were obtained from subjects and measured by researchers.

2.9. Statistical Analysis. Continuous variables and categorical variables are presented as the median (25th, 75 th percentile) or mean (SD) and counts (percentage) as appropriate. Differences between two groups were analyzed with the MannWhitney $U$ test, $t$ test, or chi-square test. Correlations between maternal data or neonatal clinical data and fetal blood test indexes were analyzed by the Spearman rank correlation test. Significance was assumed at $P<0.05$. Then, an ANCOVA analysis was performed to study the relationship between neonatal hypoglycemia and neonatal blood test by adjusting other confounders. Statistical analysis was per- formed with SPSS for Windows software (version 22, SPSS, IBM, New York, NY).

\section{Results}

3.1. Baseline Characteristics. Figure 1 showed 7289 pregnant women with fully completed blood tests in those diagnosed with GDM $^{\mathrm{a}}$ and non-GDM (1116 cases and 6173 cases, respectively) in the study. The $\mathrm{GDM}^{\mathrm{a}}$ group had tested $\mathrm{HbA1c}$ at three times to evaluate diabetic dysfunction and the prevention of delaying treatment for pregnant women. Meanwhile, the control group had the HbA1c test for three times as well.

Compared with noncases, participants who developed GDM-monitoring HbA1c levels within 5.5\%-6.4\% were more likely to be older, have higher antepartum BMI, and have higher incidence of hypoglycemia in neonates as well as premature but less gestational weeks (Table 1).

3.2. Comparison of Maternal and Fetal Blood Tests between Groups. Considering the maternal blood test levels during 
TABLE 2: Blood tests on pregnant women and neonates.

\begin{tabular}{lccc}
\hline & All groups & GDM & Control \\
\hline OGTT (mmol/L) & & & $P$ \\
Fasting blood glucose & $4.45 \pm 0.66$ & $5.35 \pm 0.91$ & $4.29 \pm 0.44$ \\
1-hour postprandial blood glucose & $7.55 \pm 1.23$ & $10.1 \pm 0.93$ & $7.09 \pm 0.50$ \\
2-hour postprandial blood glucose & $6.37 \pm 1.32$ & $8.48 \pm 1.56$ & $5.99 \pm 0.81$ \\
HbA1c (average, \%) & $4.95 \pm 0.49$ & $5.96 \pm 0.20$ & $4.76 \pm 0.25$ \\
Plasma lipid test (before delivery, mmol/L) & & & $<0.001$ \\
TG & $4.44(3.7,5.19)$ & $4.87(3.86,5.88)$ & $<.39(3.69,5.1)$ \\
TC & $6.27(5.34,7.17)$ & $6.31(5.34,7.23)$ & $6.26(5.34,7.16)$ \\
HDL-C & $2.05(1.59,2.57)$ & $2.07(1.57,2.56)$ & $2.05(1.6,2.57)$ \\
LDL-C & $2.98(2.06,3.87)$ & $2.95(2.05,3.93)$ & $2.98(2.06,3.86)$ \\
Cord blood test $(\mu M)$ & & & 0.437 \\
Gly & $7.53(1.71,308.40)$ & $15.52(2.37,346.09)$ & 0.640 \\
Isovalerylcarnitine & $0.11(0.08,0.15)$ & $0.12(0.09,0.25)$ & 0.694 \\
Octenylcarnitine & $0.07(0.05,0.10)$ & $0.08(0.05,0.14)$ & $0.67(1.63,298.50)$ \\
Palmitoylcarnitine & $1.66(1.05,2.25)$ & $1.52(0.92,2.12)$ & $0.1(0.08,0.14)$ \\
Octadecylcarnitine & $0.64(0.47,0.82)$ & $0.68(0.49,0.96)$ & $0.07(0.05,0.09)$ \\
Octadecenoylcarnitine & $0.9(0.68,1.18)$ & $0.94(0.68,1.26)$ & $1.68(1.08,2.28)$ \\
\hline Data & $0.63(0.47,0.80)$ & $<0.001$ \\
\end{tabular}

Data given as the mean \pm SD in maternal glucose profiles and as the median $\left(25^{\text {th }}, 75^{\text {th }}\right)$ in others. GDM: gestational diabetes mellitus; HbAlc: glycosylated haemoglobin; OGTT: oral glucose tolerance test; TG: triglyceride; TC: total cholesterol; HDL-C: high-density lipoprotein-cholesterol; LDL-C: low-density lipoprotein-cholesterol; Gly: glycine.

mid-late pregnancy in Table 2, all maternal blood glucose test results in the GDM group were higher than those in the nonGDM group (all $\boldsymbol{P}<0.001$ ). Meanwhile, HbAlc levels were averaged by three or four times in two groups and TG levels were slightly higher in the GDM group (4.87 vs. $4.39, \boldsymbol{P}<$ 0.001 ).

Additionally, several long-chain acylcarnitines including isovalerylcarnitine, octenylcarnitine, palmitoylcarnitine, octadecylcarnitine, and octadecenoylcarnitine were also significantly different between two groups as well as glycine (all $\boldsymbol{P}<0.05$ ). The baseline concentrations of other carnitine species and amino acids in neonatal blood are presented in Table 3.

3.3. Association between Maternal-Fetal Clinical Data and Blood Tests. Our data showed that neonatal hypoglycemia were positively correlated with GDM $(r=0.210, P<0.001)$ and HbAlc average levels $(r=0.127, P<0.001)$ but negatively correlated with gestational weeks $(r=-0.101, P<$ $0.001)$. Meanwhile, compared with the premature correlation coefficient, neonatal hypoglycemia was stronger with maternal diabetic disorder (Table 4).

Then, Table 5 summarizes the association between the maternal-fetal characteristics, and blood tests in those with significant differences were analyzed. Only GDM and $\mathrm{HbAlc}$ average levels were associated with those carnitine and glycine levels $(r=0.084, P<0.001$, and $r=0.064, P$ $<0.001$ ), while palmitoylcarnitine, octadecylcarnitine, and octadecenoylcarnitine were negative with neonatal hypoglycemia. On the other hand, fetal blood glycine, isovalerylcarnitine, and octenylcarnitine levels were all positively correlated with maternal-neonatal glycolipid dysfunction including GDM, HbAlc average levels, TG, and neonatal hypoglycemia (all $P<0.05$ ).

For further study, an ANCOVA analysis was performed to study the relationship between neonatal hypoglycemia and amino acid or carnitine levels in offspring, by adjusting related factors. Only glycine levels were significantly related with neonatal hypoglycemia and obviously elevated in umbilical plasma of neonates born to GDM mothers ( 15.52 vs. $6.67, b=0.002, P<0.001$ ) (Table 6). Meanwhile, isovalerylcarnitine and octenylcarnitine were associated with maternal TG levels but only slightly higher than the control group.

\section{Discussion}

Fetal metabolism, as indicated by carnitine levels and amino acids, may be related to the risk of preterm infants, maternal gestational age, and neonatal development [21, 22]. Dysglycemia was an important link between maternal serum amino acids and carnitines [23], maternal obesity/overweight [24], and maternal and fetal lipid profiles [25]. We explored the relationship between GDM and their neonatal carnitine levels and amino acids to figure out how to influence fetal outcomes.

Fetal development was influenced by neonatal and maternal metabolism [26, 27], and in the present study, we analyzed the effects of neonatal carnitine levels and amino acids on neonatal growth parameters. We found that cord blood glycine levels were different between two groups and correlated with maternal glucolipid when $\mathrm{HbAlc}$ levels were between 5.5\% and 6.4\%. Meanwhile, glycine levels in umbilical cord blood were also correlated with neonatal glucose dysfunction. 
TABLE 3: Blood tests on pregnant women and neonates.

\begin{tabular}{|c|c|c|c|c|}
\hline & All groups & GDM & Control & $P$ \\
\hline HbA1c (24-28 weeks, \%) & $4.97 \pm 0.69$ & $6.15 \pm 0.17$ & $4.76 \pm 0.52$ & $<0.001$ \\
\hline HbA1c (28-32 weeks, \%) & $5.08 \pm 0.72$ & $6.15 \pm 0.17$ & $4.69 \pm 0.35$ & $<0.001$ \\
\hline HbA1c (32-36 weeks, \%) & $4.94 \pm 0.48$ & $5.53 \pm 0.78$ & $4.8 \pm 0.2$ & $<0.001$ \\
\hline HbA1c (average before delivery, \%) & $4.93 \pm 0.78$ & $5.91 \pm 1.17$ & $4.75 \pm 0.52$ & $<0.001$ \\
\hline \multicolumn{5}{|l|}{ Cord blood test $(\mu \mathrm{M})$} \\
\hline Ala & $214.16(160.26,301.23)$ & $212.91(160.3,301.55)$ & $214.33(160.23,301.01)$ & 0.824 \\
\hline Arg & $2.06(1.34,3.5)$ & $2(1.38,3.58)$ & $2.07(1.33,3.47)$ & 0.356 \\
\hline Asp & $27.95(19.38,38.45)$ & $28.1(19.51,39.22)$ & $27.92(19.36,38.33)$ & 0.717 \\
\hline Cit & $9.04(7.35,11.12)$ & $9.05(7.3,11.06)$ & $9.04(7.35,11.13)$ & 0.962 \\
\hline Gln & $2.52(1.39,7.67)$ & $2.55(1.41,7.52)$ & $2.51(1.38,7.69)$ & 0.828 \\
\hline Glu & $30.31(2.29,392.88)$ & $42.51(2.25,405.86)$ & $29.5(2.3,387.7)$ & 0.669 \\
\hline His & $376.68(218.92,536.89)$ & $379.85(218.63,535.29)$ & $376.19(218.92,537.25)$ & 0.816 \\
\hline Leu & $314.34(125.43,456.15)$ & $301.37(112.69,456.96)$ & $316.4(128.63,456.02)$ & 0.056 \\
\hline Met & $28.43(19.95,39.82)$ & $27.87(19.38,39.51)$ & $28.5(20.05,39.9)$ & 0.463 \\
\hline Orn & $87.64(61.97,111.26)$ & $85.71(60.1,110.02)$ & $87.92(62.61,111.36)$ & 0.184 \\
\hline Phe & $27.07(21.12,40.02)$ & $27.66(21.29,43.02)$ & $26.99(21.08,39.43)$ & 0.142 \\
\hline Ser & $61.43(45.07,87.31)$ & $63.63(46.22,89.01)$ & $61.03(44.85,86.95)$ & 0.054 \\
\hline Thr & $49(31.95,67.83)$ & $48.9(32.25,66.37)$ & $49.01(31.92,68.14)$ & 0.926 \\
\hline $\operatorname{Trp}$ & $85.6(62.89,109.61)$ & $84.87(62.39,110.19)$ & $85.66(63.05,109.47)$ & 0.560 \\
\hline Tyr & $48.23(34.32,61.86)$ & $48.81(34.03,62.44)$ & $48.11(34.38,61.74)$ & 0.343 \\
\hline Val & $56.06(42.67,71.32)$ & $56.79(43.14,71.62)$ & $55.79(42.61,71.23)$ & 0.157 \\
\hline Free carnitine & $54.75(38.34,71.5)$ & $54.84(38.07,73.18)$ & $54.72(38.42,71.22)$ & 0.962 \\
\hline Acetylcarnitine & $30.7(20.81,40.73)$ & $29.96(19.94,40.09)$ & $30.88(20.99,40.89)$ & 0.147 \\
\hline Propionylcarnitine & $4.22(1.82,19.04)$ & $4.22(1.9,18.43)$ & $4.22(1.81,19.15)$ & 0.986 \\
\hline Malonylcarnitine & $0.05(0.04,0.13)$ & $0.05(0.04,0.13)$ & $0.05(0.04,0.13)$ & 0.975 \\
\hline Butylcarnitine & $0.13(0.09,0.18)$ & $0.13(0.09,0.18)$ & $0.13(0.09,0.18)$ & 0.661 \\
\hline Succinylcarnitine & $0.19(0.14,0.26)$ & $0.19(0.15,0.26)$ & $0.19(0.14,0.26)$ & 0.847 \\
\hline 3-Hydroxyisobutyryl-carnitine & $0.1(0.07,0.16)$ & $0.11(0.07,0.16)$ & $0.1(0.07,0.15)$ & 0.606 \\
\hline Senecioylcarnitine & $0.03(0.02,0.03)$ & $0.03(0.02,0.03)$ & $0.03(0.02,0.03)$ & 0.448 \\
\hline Glutarylcarnitine & $0.03(0.02,0.05)$ & $0.03(0.02,0.05)$ & $0.03(0.02,0.05)$ & 0.596 \\
\hline 3-Hydroxyisovaleryl-carnitine & $0.14(0.11,0.17)$ & $0.13(0.11,0.17)$ & $0.14(0.11,0.17)$ & 0.849 \\
\hline Caproylcarnitine & $0.02(0.01,0.03)$ & $0.02(0.01,0.03)$ & $0.02(0.01,0.03)$ & 0.964 \\
\hline Hexenoylcarnitine & $0.01(0.01,0.01)$ & $0.01(0.01,0.01)$ & $0.01(0.01,0.01)$ & 0.059 \\
\hline Adipoylcarnitine & $0.03(0.02,0.04)$ & $0.03(0.02,0.04)$ & $0.03(0.02,0.04)$ & 0.341 \\
\hline Decoylcarnitine & $0.04(0.02,0.06)$ & $0.04(0.02,0.06)$ & $0.04(0.02,0.06)$ & 0.363 \\
\hline Octanedioylcarnitine & $0.02(0.01,0.02)$ & $0.02(0.01,0.02)$ & $0.02(0.01,0.02)$ & 0.421 \\
\hline Decanoylcarnitine & $0.04(0.02,0.07)$ & $0.04(0.02,0.07)$ & $0.04(0.02,0.07)$ & 0.579 \\
\hline Alkaloidcarnitine & $0.04(0.02,0.06)$ & $0.04(0.02,0.06)$ & $0.04(0.02,0.06)$ & 0.102 \\
\hline Lauroylcarnitine & $0.06(0.04,0.09)$ & $0.06(0.04,0.1)$ & $0.06(0.04,0.09)$ & 0.199 \\
\hline Myrcenecarnitine & $0.02(0.01,0.03)$ & $0.02(0.01,0.03)$ & $0.02(0.01,0.03)$ & 0.410 \\
\hline Myristoylcarnitine & $0.14(0.11,0.19)$ & $0.15(0.11,0.19)$ & $0.14(0.11,0.19)$ & 0.591 \\
\hline Alkenyl-myristoyl-carnitine & $0.05(0.03,0.08)$ & $0.05(0.03,0.08)$ & $0.05(0.03,0.08)$ & 0.226 \\
\hline 3-Hydroxy-myristoyl-carnitine & $0.01(0.01,0.02)$ & $0.01(0.01,0.01)$ & $0.01(0.01,0.02)$ & 0.282 \\
\hline Palmitoyl-enoyl-carnitine & $0.09(0.05,0.13)$ & $0.09(0.05,0.13)$ & $0.09(0.05,0.13)$ & 0.877 \\
\hline 3-Hydroxy-palmitoyl-carnitine & $0.01(0.01,0.02)$ & $0.01(0.01,0.02)$ & $0.01(0.01,0.02)$ & 0.428 \\
\hline 3-Hydroxy-octadecanoyl-carnitine & $0.01(0.01,0.01)$ & $0.01(0.01,0.01)$ & $0.01(0.01,0.01)$ & 0.756 \\
\hline
\end{tabular}

Data given as the mean \pm SD in maternal glucose profiles and as the median $\left(25^{\text {th }}, 75^{\text {th }}\right)$ in others. GDM: gestational diabetes mellitus; HbAlc: glycosylated haemoglobin; Ala: alanine; Arg: arginine; Asp: aspartic acid; Cit: citrulline; Gln: glutamine; Glu: glutamic acid; His: histidine; Leu: leucine; Met: methionine; Orn: ornithine; Phe: phenylalanine; Ser: serine; Thr: threonine; Trp: tryptophan; Tyr: tyrosine; Val: valine. 
TABLE 4: Correlations between neonatal outcomes and maternal characteristics.

\begin{tabular}{lcccc}
\hline & \multicolumn{2}{c}{ Neonatal } & \multicolumn{2}{c}{ Premature } \\
& $r$ & $P$ & $r$ & $P$ \\
\hline GDM & 0.210 & $<0.001$ & 0.125 & $<0.001$ \\
Maternal age & 0.017 & 0.139 & 0.014 & 0.243 \\
Gestational weeks & -0.101 & $<0.001$ & -0.449 & $<0.001$ \\
Antepartum BMI & -0.019 & 0.107 & -0.025 & 0.032 \\
Maternal HbA1c (average) & 0.127 & $<0.001$ & 0.079 & $<0.001$ \\
TG & 0.020 & 0.092 & 0.014 & 0.233 \\
\hline
\end{tabular}

GDM: gestational diabetes mellitus; BMI: body mass index; HbAlc: glycosylated haemoglobin; TG: triglyceride.

The association between maternal glycolipid metabolism and neonatal development had been reported in previous studies [28], and our results supported previous studies in a larger study population. There were 17 types of amino acids and 30 types of carnitines in cord blood to be analyzed, and it was the first original study to fully analyze those neonatal amino acid and carnitine metabolisms compared with previous studies [29]. However, the negative associations were between carnitine levels and neonatal hypoglycemia. The results in the present study not only found that glycine levels had an independently positive relationship with neonatal hypoglycemia but also firstly revealed the association between neonatal hypoglycemia and neonatal glycine levels with maternal glucose dysfunction in mid-late gestation in a new research population.

The result of the present study mainly showed that, although neonatal hypoglycemia was positively correlated with glycine levels in umbilical cord blood, it had a pretty low effect size to hypoglycemia in offspring. Unfortunately, glycine levels were probably not an effective biomarker for predicting the risk of neonatal hypoglycemia. Meanwhile, these findings also implicitly expressed that neonatal hypoglycemia was influenced by multi-maternal-neonatal factors and an extremely complicated multifactor, multivariable, and multilevel dynamic process and even multisystems, especially placenta.

Additionally, it was clearly proven that the incidence of adverse neonatal outcomes was higher when $\mathrm{HbAlc}$ levels ranged 5.5\% 6.4\% among pregnant women in GDM, and GDM can affect neonatal amino acid and carnitine levels. It was the first research to reveal how to influence fetal outcomes and specific amino acid and carnitine levels in offspring. It ineffectively predicted the risk of hypoglycemia for newborns, but it was confidently involved in the biological process on neonatal hypoglycemia.

There was no doubt that amino acid is the basic unit of protein and is involved in the entire glycolipid process during pregnancy and neonatal development in many studies from human beings or animals [30,31]. Meanwhile, the amino acid amount can affect amount important signaling molecules and natural products during biological metabolism, then affect synthesis of most proteins involved in maternal- neonatal metabolism. However, it was too difficult to figure out which were key points to determine obvious adverse outcomes between mothers and neonates because of clinical interferences and lack of advanced animal experiments data, especially pregnancy researches.

Objectively, glycine levels were obviously higher than in the control group, but it was hard to explain the original biological resources and why it is higher because the umbilical cord blood was mixed with maternal and fetal blood. However, the first large prospective case-cohort observational study was a confident evidence to reflect real-time perspective on maternal-neonatal metabolic changes after the delivery. Meanwhile, glycine may be a potential biomarker to trace glucose metabolism for further study during prenatal development since the significant difference of glycine levels was higher in maternal glucose metabolism dysfunction and an independent factor to affect neonatal hypoglycemia as well.

Some studies showed that plasma glycine positively correlated with glucose disposal, and dietary glycine supplementation increases insulin [32]. Glycine levels rose higher in the GDM group than in the control group, and the elevation of insulin levels may be followed to contribute to neonatal hypoglycemia. However, it was unclear whether neonatal hypoglycemia was associated with self-insulin levels by the influence of glycine levels and whether glycine levels truly influenced glucose disposal during labor through the placenta. It needs more specific biological experiments and deserves further study to prevent adverse outcomes in offspring.

Recently, the crosstalk between GDM and fetal growth is well established [33]. During pregnancy, dysglycemia is typically accompanied by dyslipidemia [34], and together, they promoted an adverse metabolic intrauterine environment and led to preterm infants [35] or congenital malformations [36]. GDM is strongly correlated with fetal growth [37], but neonatal hypoglycemia was negative with different fetal carnitine levels in our study. Most studies reported that carnitine was associated with the development and progress of diabetes mellitus for multiperspectives such as microbiota [38] and lipid [39].

Besides, in our study, there were some significant differences between groups for the measured carnitine parameters in newborns and positively correlated with maternal glucose metabolism dysfunction. Meanwhile, isovalerylcarnitine and octenylcarnitine were positively correlated with GDM with glycosylated haemoglobin levels of 5.5\%-6.4\% but with low effective size the same as glycine. However, neonatal carnitine levels were not associated with their hypoglycemia in the present study.

There are several limitations in the present study. First, although the present study provided insight into the association between maternal glycolipid factors and neonatal metabolism and characteristic size, the observational design could be another limitation because of the lack of effective size for the prediction. The principle of "equilibrium" was mainly for aiming at maintaining high statistical efficiency in researches. However, it strongly ensured that the research object was close to the real world and reflected the real 


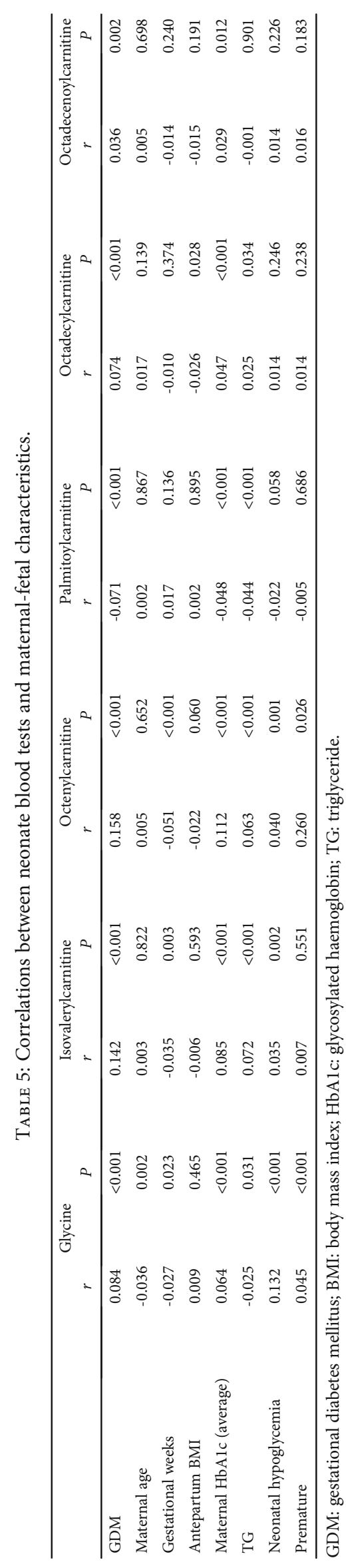


TABLE 6: The relationship between neonatal hypoglycemia and umbilical cord blood of amino acid and carnitine via ANCOVA analysis.

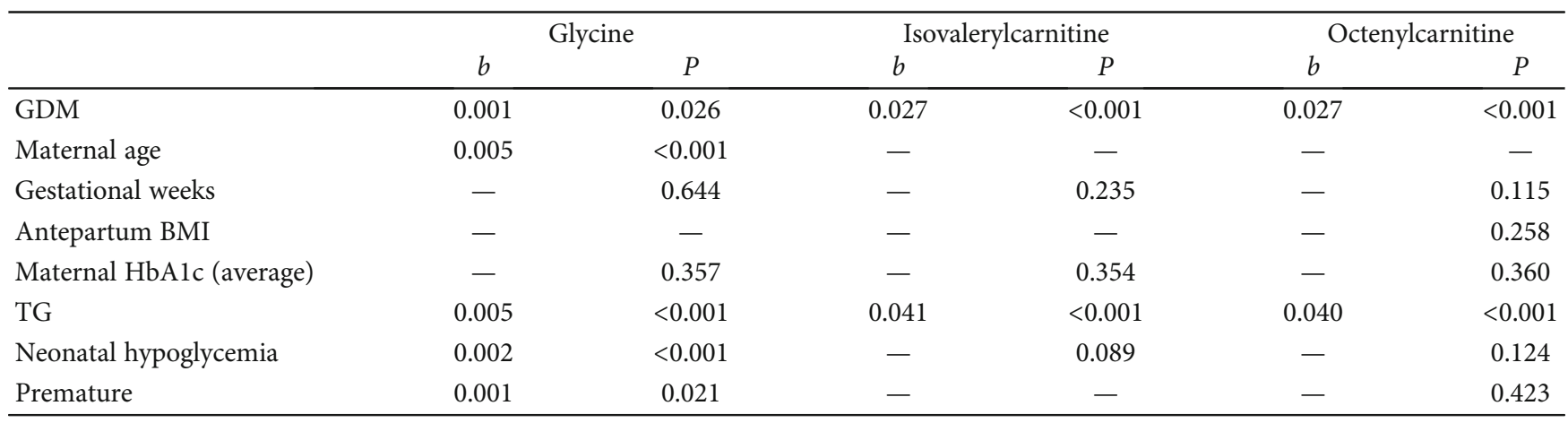

GDM: gestational diabetes mellitus; BMI: body mass index; HbA1c: glycosylated haemoglobin; TG: triglyceride.

relationship in clinical phenomenon, especially observational studies and diagnostic accuracy tests [40].

Meanwhile, it was still unclear that how neonatal hypoglycemia was influenced by metabolism changes after delivery. The leading explanation was that maternal insulin levels were delivered to the baby or the neonatal liver produced higher insulin levels in GDM. However, it could not explain why the higher incidence of neonatal hypoglycemia was in preterm neonates [41].

Additionally, since insulin levels of cord blood in the present study were not checked in the two groups, the relationship between glycine and the function of insulin including C-peptide was unclear. Neonatal insulin and C-peptide levels were inaccurately analyzed by the normal clinical standard, as well as glycosylated haemoglobin for newborns [42].

Furthermore, variations in lipid concentration were considerable during gestation, but we only obtained lipid values in late gestation. Therefore, further prospective studies measuring maternal glycolipid concentrations across multiple gestational times in a wide-ranging population were needed to increase the validity of the results.

\section{Conclusion}

In summary, our results showed that glycine levels in cord blood were independently positive with neonatal hypoglycemia in maternal glucose dysfunction when pregnant women in GDM fluctuating HbA1c levels ranging from 5.5\% to $6.4 \%$ during mid-late pregnancy, compared to the non-GDM group. Meanwhile, glycine levels have a positive relationship with maternal-neonatal diabetic outcomes, but lack of effective size to predict neonatal hypoglycemia. Glycine may be a potential biomarker as the key departure to trace the underlying mechanisms of neonatal hypoglycemia.

\section{Data Availability}

We can provide the data with permission from three official centers (The Fifth Affiliated Hospital of Sun Yat-sen University, Zhuhai Center for Maternal and Child Health Care, and Zhuhai People's Hospital).

\section{Ethical Approval}

The study protocol was established, according to the ethical guidelines of the Helsinki Declaration, and was approved by the ethical committee of the Fifth Affiliated Hospital of Sun Yat-sen University.

\section{Consent}

Written informed consent was obtained from individual or guardian participants.

\section{Disclosure}

The funding organisation had no role in the study design, data collection, analysis, interpretation, or writing of the manuscript.

\section{Conflicts of Interest}

The authors declare that they have no competing interests. No conflicts of interest that could be perceived as prejudicing the impartiality of the research are reported.

\section{Authors' Contributions}

YL and ZS designed the experiments; YY, WL, and LC carried out the experiments; and YL, HZ, and WC analyzed the experimental results. YY, YL, LC, and WL analyzed the sequencing data and developed the analysis tools. FJ, TH, JW, YC, and JC made a series of inclusions and follow-ups of patients. DX randomly extracted samples to match. YL wrote the manuscript.

\section{Acknowledgments}

We appreciated Ran Xue for the analysis of the cord blood test in the manuscript. This project was funded by the Science and Technology Project of China, Zhuhai (to ZS).

\section{References}

[1] M. V. A. H. Senat, A. Letourneau, M. Coustols-Valat, M. Cazaubiel, and H. Legardeur, "Effect of glyburide vs 
subcutaneous insulin on perinatal complications among women with gestational diabetes," Journal of the American Medical Association, vol. 319, no. 17, pp. 1773-1780, 2018.

[2] E. A. B. Chiefari, D. Foti, and A. Brunetti, "Gestational diabetes mellitus: an updated overview," Journal of Endocrinological Investigation, vol. 40, no. 9, pp. 899-909, 2017.

[3] J. M. B. L. Gerome and G. Dogbey, "Effects of implementing International Association of Diabetes and Pregnancy Study Groups gestational diabetes screening on pregnancy outcomes at a small community teaching hospital," Clinical Diabetes, vol. 35, no. 2, pp. 84-89, 2017.

[4] L. P. M. B. Lowe, A. R. Dyer, J. Lowe, D. R. McCance, and T. R. Lappin, "Hyperglycemia and Adverse Pregnancy Outcome (HAPO) study: associations of maternal A1C and glucose with pregnancy outcomes," Diabetes Care, vol. 35, no. 3, pp. 574580, 2012.

[5] B. Bhavadharini, M. M. Mahalakshmi, R. M. Anjana et al., "Prevalence of gestational diabetes mellitus in urban and rural Tamil Nadu using IADPSG and WHO 1999 criteria (WINGS 6)," Clinical Diabetes and Endocrinology, vol. 2, no. 8, pp. 16$28,2016$.

[6] C. W. Y. Wang, X. Zhang, Y. Zhang, Q. Xu, and Y. Sun, "A randomized clinical trial of exercise during pregnancy to prevent gestational diabetes mellitus and improve pregnancy outcome in overweight and obese pregnant women," American Journal of Obstetrics and Gynecology, vol. 216, no. 4, pp. 340-351, 2017.

[7] J. M. A. Tieu, C. A. Crowther, P. Middleton, and E. Shepherd, "Screening for gestational diabetes mellitus based on different risk profiles and settings for improving maternal and infant health," Cochrane Database of Systematic Reviews, vol. 8, article CD007222, 2017.

[8] M. S.-A. G. Adeva-Andany, E. Ameneiros-Rodriguez, C. Fernandez-Fernandez, C. Donapetry-Garcia, and A. Dominguez-Montero, "Insulin resistance and glycine metabolism in humans," Amino Acids, vol. 50, no. 1, pp. 1127, 2018.

[9] E. R. E. Strand, M. R. Flygel, V. Lysne, G. F. T. Svingen, and G. S. Tell, "Serum carnitine metabolites and incident type 2 diabetes mellitus in patients with suspected stable angina pectoris," The Journal of Clinical Endocrinology and Metabolism, vol. 103, no. 3, pp. 1033-1041, 2018.

[10] D. L. Ackerman, K. M. Craft, and S. D. Townsend, "Infant food applications of complex carbohydrates: structure, synthesis, and function," Carbohydrate Research, vol. 437, pp. 16-27, 2017.

[11] A. A. Geraghty, G. Alberdi, E. J. O’Sullivan et al., "Maternal and fetal blood lipid concentrations during pregnancy differ by maternal body mass index: findings from the ROLO study," BMC Pregnancy and Childbirth, vol. 17, no. 1, p. 360, 2017.

[12] G. Tajima, K. Hara, M. Tsumura et al., "Newborn screening for carnitine palmitoyltransferase II deficiency using (C16 + C18:1)/C2: evaluation of additional indices for adequate sensitivity and lower false-positivity," Molecular Genetics and Metabolism, vol. 122, no. 3, pp. 67-75, 2017.

[13] G. Liu, W. Deng, W. Cui et al., "Analysis of amino acid and acyl carnitine profiles in maternal and fetal serum from preeclampsia patients," The Journal of Maternal-Fetal \& Neonatal Medicine, vol. 33, pp. 2743-2750, 2018.

[14] P. D. Manta-Vogli, K. H. Schulpis, Y. Dotsikas, and Y. L. Loukas, "The significant role of amino acids during pregnancy: nutritional support," The Journal of Maternal-Fetal \& Neonatal Medicine, vol. 33, pp. 334-340, 2018.

[15] A. Edward, D. W. B. Liechty, L. A. HM, and S. C. Denne, "Aromatic amino acids are utilized and protein synthesis is stimulated during amino acid infusion in the ovine fetus," Nutrient Metabolism, vol. 129, no. 6, pp. 1161-1166, 1999.

[16] A. E. P. Heazell, G. Bernatavicius, L. Warrander, M. C. Brown, and W. B. Dunn, "A metabolomic approach identifies differences in maternal serum in third trimester pregnancies that end in poor perinatal outcome," Reproductive Sciences, vol. 19, no. 8, pp. 863-875, 2012.

[17] S. M. T. Huang, T. Norris, S. Sun, T. Zhang, and T. L. Han, "The CLIMB (Complex Lipids In Mothers and Babies) study: protocol for a multicentre, three-group, parallel randomised controlled trial to investigate the effect of supplementation of complex lipids in pregnancy, on maternal ganglioside status and subsequent cognitive outcomes in the offspring," $B M J$ Open, vol. 7, no. 10, article e016637, 2017.

[18] M. Azar, J. A. Stoner, H. D. Dao et al., "Epidemiology of dysglycemia in pregnant Oklahoma American Indian women," The Journal of Clinical Endocrinology and Metabolism, vol. 100, no. 8, pp. 2996-3003, 2015.

[19] K. Benhalima, P. Van Crombrugge, C. Moyson et al., "Characteristics and pregnancy outcomes across gestational diabetes mellitus subtypes based on insulin resistance," Diabetologia, vol. 62, no. 11, pp. 2118-2128, 2019.

[20] Committee on Fetus and Newborn, "Postnatal glucose homeostasis in late-preterm and term infants," Pediatrics, vol. 127, no. 3, pp. 575-579, 2011.

[21] F. Bonsante, J.-B. Gouyon, P.-Y. Robillard, B. Gouyon, and S. Iacobelli, "Early optimal parenteral nutrition and metabolic acidosis in very preterm infants," PLoS One, vol. 12, no. 11, article e0186936, 2017.

[22] M. A. Clark, R. E. K. Stein, E. J. Silver, S. Khalid, M. Fuloria, and N. V. Esteban-Cruciani, "Carnitine deficiency in preterm infants: a national survey of knowledge and practices," Journal of Neonatal-Perinatal Medicine, vol. 10, no. 4, pp. 381-386, 2017.

[23] J. Nevalainen, M. Sairanen, H. Appelblom, M. Gissler, S. Timonen, and M. Ryynänen, "First-trimester maternal serum amino acids and acylcarnitines are significant predictors of gestational diabetes," The Review of Diabetic Studies, vol. 13, no. 4, pp. 236-245, 2016.

[24] L. R. B. H. De Souza, R. Retnakaran, J. L. Maguire, A. B. Nathens, and P. W. Connelly, "First-trimester maternal abdominal adiposity predicts dysglycemia and gestational diabetes mellitus in midpregnancy," Diabetes Care, vol. 39, no. 1, pp. 61-64, 2016.

[25] E. Herrera and G. Desoye, "Maternal and fetal lipid metabolism under normal and gestational diabetic conditions," Hormone Molecular Biology and Clinical Investigation, vol. 26, no. 2, pp. 109-127, 2016.

[26] H. Akkaya, B. Büke, and G. Uysal, "Fetal pancreatic hyperechogenicity may be an early ultrasonographic sign of gestational diabetes mellitus," The Journal of Maternal-Fetal \& Neonatal Medicine, vol. 33, pp. 2387-2394, 2018.

[27] L. A. Barbour and T. L. Hernandez, "Maternal lipids and fetal overgrowth: making fat from fat," Clinical Therapeutics, vol. 40, no. 10, pp. 1638-1647, 2018.

[28] W. L. Lowe, D. M. Scholtens, L. P. Lowe et al., "Association of gestational diabetes with maternal disorders of glucose 
metabolism and childhood adiposity," Journal of the American Medical Association, vol. 320, no. 10, pp. 1005-1016, 2018.

[29] W. L. Lowe, J. R. Bain, M. Nodzenski et al., "Maternal BMI and glycemia impact the fetal metabolome," Diabetes Care, vol. 40, no. 7, pp. 902-910, 2017.

[30] C. S. Yost, C. D. Lopez, S. B. Prusiner, R. M. Myers, and V. R. Lingappa, "Non-hydrophobic extracytoplasmic determinant of stop transfer in the prion protein," Nature, vol. 343, no. 6259, pp. 669-672, 1990.

[31] Y. Zhao, Z. Bao, Z. Wan, Z. Fu, and Y. Jin, "Polystyrene microplastic exposure disturbs hepatic glycolipid metabolism at the physiological, biochemical, and transcriptomic levels in adult zebrafish," Science of The Total Environment, vol. 710, no. 136279, p. 27, 2020.

[32] R. Yan-Do and P. E. MacDonald, "Impaired "glycine"-mia in type 2 diabetes and potential mechanisms contributing to glucose homeostasis," Endocrinology, vol. 158, no. 5, pp. 10641073, 2017.

[33] R. F. Goldstein, S. K. Abell, S. Ranasinha et al., "Association of Gestational Weight Gain with Maternal and Infant Outcomes: a systematic review and meta-analysis," Journal of the American Medical Association, vol. 317, no. 21, pp. 2207-2225, 2017.

[34] A. P. O. R. Herrera Martinez, R. Bahamondes Opazo, and P. Moreno-Moreno, "Hyperlipidemia during gestational diabetes and its relation with maternal and offspring complications," Nutrición Hospitalaria, vol. 35, no. 3, pp. 698-706, 2018.

[35] W. H. W. Zheng, L. Zhang, Z. Tian, T. Wang, and T. Zhang, "Changes in serum lipid levels during pregnancy and association with neonatal outcomes: a large cohort study," Reproductive Sciences, vol. 25, no. 9, pp. 1406-1412, 2018.

[36] L. Bequer, T. Gómez, J. L. Molina, A. Álvarez, C. Chaviano, and S. Clapés, "Experimental diabetes impairs maternal reproductive performance in pregnant Wistar rats and their offspring," Systems Biology in Reproductive Medicine, vol. 64, no. 1, pp. 60-70, 2018.

[37] E. C. Johns, F. C. Denison, J. E. Norman, and R. M. Reynolds, "Gestational diabetes mellitus: mechanisms, treatment, and complications," Trends in Endocrinology and Metabolism, vol. 29, no. 11, pp. 743-754, 2018.

[38] Y. Heianza, D. Sun, X. Li et al., "Gut microbiota metabolites, amino acid metabolites and improvements in insulin sensitivity and glucose metabolism: the POUNDS Lost trial," Gut, vol. 68, no. 2, pp. 263-270, 2019.

[39] M. Prentki, B. E. Corkey, and S. R. M. Madiraju, "Lipid-associated metabolic signalling networks in pancreatic beta cell function," Diabetologia, vol. 63, no. 1, pp. 10-20, 2020.

[40] V. A. Benites-Zapata, A. V. Hernandez, V. Nagarajan, C. A. Cauthen, R. C. Starling, and W. H. W. Tang, "Usefulness of neutrophil-to-lymphocyte ratio in risk stratification of patients with advanced heart failure," The American Journal of Cardiology, vol. 115, no. 1, pp. 57-61, 2015.

[41] Y. Y. Sun, J. Juan, Q. Q. Xu, R. N. Su, J. E. Hirst, and H. X. Yang, "Increasing insulin resistance predicts adverse pregnancy outcomes in women with gestational diabetes mellitus," Journal of Diabetes, vol. 12, no. 6, pp. 438-446, 2020.

[42] J.-H. Sung, D. Y. Lee, K. P. Min, and C.-Y. Park, "Peripartum management of gestational diabetes using a digital health care service: a pilot, randomized controlled study," Clinical Therapeutics, vol. 41, no. 11, pp. 2426-2434, 2019. 